Dochead pratique

Sous-dochead pharmacovigilance

\title{
Les interactions pharmacocinétiques et pharmacodynamiques
}

\author{
Hélène Geniaux ${ }^{\mathrm{a}, \mathrm{b}}$ \\ Praticien hospitalier \\ Nicolas Picard $\mathbf{d}^{\mathrm{b}, *, \mathrm{c}}$ \\ Professeur des Universités, praticien hospitalier
}

${ }^{a}$ Centre régional de pharmacovigilance, de pharmaco-épidémiologie et d'information sur les médicaments. Service de pharmacologie, toxicologie et pharmacovigilance, 2 avenue Martin-LutherKing, 87042 Limoges cedex, France

${ }^{\mathrm{b}} \mathrm{Centre}$ hospitalier universitaire de Limoges, Service de pharmacologie, toxicologie et pharmacovigilance, 2 avenue Martin-Luther-King, 87042 Limoges cedex, France

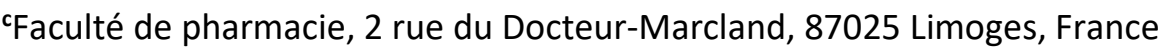

*Auteur correspondant.

Adresse e-mail : nicolas.picard@unilim.fr (N. Picard).

\section{Résumé}

Les interactions médicamenteuses relèvent de deux mécanismes : pharmacocinétique et pharmacodynamique. Minimiser le risque d'interactions délétères passe par une bonne connaissance de la pharmacologie des médicaments, que ceux-ci soient disponibles sur prescription ou en automédication.

(C) 2019

Mots clés - cytochrome P450 ; interaction médicamenteuse ; pharmacocinétique ; pharmacodynamie

Summary à venir

(C) 2019

Keywords à venir 
Parmi les interactions médicamenteuses, celles impliquant une transformation de la pharmacocinétique d'un médicament "victime" d'une seconde molécule, "coupable" de l'interaction, sont les plus nombreuses. Elles résultent d'une modification d'une des étapes de la pharmacocinétique du premier médicament (absorption, distribution, métabolisme ou élimination), susceptible de faire varier sa concentration sanguine. Il est question d'interaction pharmacodynamique quand l'effet ou la "réponse" d'un médicament est modifié en présence d'un (ou plusieurs) autre(s), sans changement de leurs concentrations plasmatiques.

\section{T1 Les interactions pharmacocinétiques}

TEG1 Les interactions les plus préjudiciables sont celles qui amènent la concentration sanguine du médicament en dehors de l'intervalle thérapeutique (intervalle entre la concentration minimale efficace et la concentration maximale tolérée du médicament). Plus cet intervalle est large, moins l'interaction est problématique.

TEG1 Sur le plan mécanistique, l'étape de métabolisme du médicament est la plus concernée par les interactions. Pour de nombreuses molécules, cette étape de biotransformation enzymatique amorce la phase d'élimination. Les enzymes les plus impliquées sont les cytochromes P450 qui prennent en charge près de $75 \%$ des médicaments à métabolisme hépatique. Parmi eux, le cytochrome 3A4 serait en cause dans plus d'une réaction sur deux. Il est exprimé au niveau hépatique, mais aussi intestinal.

\section{T2 Induction et inhibition enzymatiques}

TEG1 L'activité des enzymes du métabolisme des médicaments peut être augmentée (c'est l'induction enzymatique) ou diminuée ( $c$ 'est l'inhibition enzymatique). L'induction accélère l'élimination du médicament victime alors que l'inhibition la ralentit. L'identification des inducteurs ou des inhibiteurs enzymatiques est donc cruciale au moment de la prescription ou lors de l'analyse pharmaceutique.

TEG1 Il convient de se référer aux résumés des caractéristiques des produits (RCP) et au Thesaurus des interactions médicamenteuses pour connaître le niveau de risque d'une interaction et rassembler plus d'éléments sur son mécanisme. Accessibles librement via la base de données publique des médicaments [1] et le Thesaurus de l'Agence nationale du médicament et des produits de santé (ANSM) [2], les RCP sont des outils indispensables. Le Thesaurus regroupe toutes les interactions importantes, quels que soient leurs mécanismes. Pour y être mentionnée, une interaction doit avoir une traduction clinique significative, décrite ou potentiellement grave, c'est-àdire susceptible de provoquer ou majorer des effets indésirables ou d'entraîner, par réduction de l'activité, une moindre efficacité des traitements.

TEG1 Le site internet des Hôpitaux Universitaires de Genève (HUG) propose, pour sa part, un document synthétique et régulièrement mis à jour qui peut être recommandé en complément des sources institutionnelles [3]. Ce dernier liste les principaux inducteurs et inhibiteurs des cytochromes P450 et d'une protéine d'efflux, la P-glycoprotéine. Les inhibiteurs répertoriés sont beaucoup plus nombreux que les inducteurs ( 25 inducteurs seulement dont 15 puissants). La liste inclut des 
substances non médicamenteuses (les goudrons du tabac, inducteurs du cytochrome $1 \mathrm{~A} 2$; l'éthanol, inducteur du cytochrome 2E1) et un produit de phytothérapie bien connu, le millepertuis.

Ce document permet aussi de retrouver les enzymes impliquées dans le métabolisme d'une liste relativement exhaustive de médicaments. Dans l'analyse d'une interaction, il est en effet crucial de connaître la voie d'élimination du médicament potentiellement victime. À titre d'exemple, l'érythromycine (inhibiteur puissant du cytochrome 3A4) est déconseillée chez un patient traité par tacrolimus car ce dernier est métabolisé par le cytochrome 3A4. En revanche, la ciprofloxacine, inhibiteur puissant du cytochrome $1 \mathrm{~A} 2$ (mais pas du 3A4), peut être utilisée sans risque car le cytochrome $1 \mathrm{~A} 2$ ne participe pas à l'élimination du tacrolimus.

\section{T2 Autres interactions pharmacocinétiques}

TEG1 Les interactions avec les cytochromes ne sont pas les seules qu'il convient de savoir reconnaître. Les antiacides (topiques ou inhibiteurs de la pompe à protons [IPP]) peuvent être à I'origine d'interactions majeures concernant la phase d'absorption. L'acidité gastrique participe à la solubilisation de certaines molécules ou leur permet de franchir la paroi digestive. Ainsi, la prise conjointe d'IPP et d'atazanavir est déconseillée : les concentrations sanguines de l'antirétroviral peuvent être réduites drastiquement [4].

TEG1 Au niveau rénal, les médicaments réduisant le débit de filtration glomérulaire (DFG), tels les anti-inflammatoires non stéroïdiens (AINS), sont susceptibles d'augmenter la concentration sanguine de ceux éliminés par filtration dans les urines (metformine par exemple).

\section{T2 Gestion des interactions pharmacocinétiques}

TEG1 La gestion d'une interaction pharmacocinétique est souvent possible, sauf lorsqu'elle relève d'une contre-indication. Il est, par exemple, envisageable de modifier la dose administrée du médicament victime pour compenser l'effet d'une inhibition ou d'une induction enzymatique. Cette adaptation posologique doit toujours être guidée par des éléments cliniques ou biologiques, idéalement une mesure des concentrations sanguines du médicament, en particulier quand la marge thérapeutique est étroite.

TEG1 Le profil physiopathologique du patient constitue le dernier point à considérer dans la gestion d'une interaction de nature pharmacocinétique. L'activité des enzymes du métabolisme, de même que le DFG, diminue avec l'âge. L'impact d'un inhibiteur enzymatique sera d'autant plus fort dans cette population qui est, par ailleurs, la plus concernée par la polymédication.

TEG1 Au final, le verdict du professionnel de santé concernant une interaction doit s'appuyer sur une analyse de la pharmacocinétique du médicament victime et l'estimation des préjudices encourus en cas d'association. Il doit être mesuré : il ne faut pas forcément renoncer à une association qui pourrait présenter un bénéfice thérapeutique.

\section{T1 Les interactions pharmacodynamiques}


TEG1 Les interactions pharmacodynamiques concernent des médicaments ayant des propriétés pharmacologiques communes, complémentaires ou antagonistes, ou encore des effets indésirables communs ou opposés vis-à-vis d'un même système physiologique. Ainsi, les interactions par potentialisation, synergie ou antagonisme sont distinguées.

TEG1 Ces interactions ont des conséquences cliniques parfois graves, mais relativement prévisibles sur la base des connaissances des propriétés pharmacologiques. Elles sont donc a priori évitables ou gérables, sous couvert d'une surveillance clinique ou d'une adaptation de la posologie.

\section{T2 Les types d'interactions}

Différents types d'interactions sont répertoriés :

- au niveau d'un même système, sur lequel les deux médicaments peuvent exercer des effets identiques ou opposés (exemple des sartans et des inhibiteurs de l'enzyme de conversion [IEC] au niveau du système rénine-angiotensine) ;

- au niveau d'une même cible cellulaire, sur laquelle les deux médicaments peuvent exercer des effets identiques ou opposés, comme les récepteurs morphiniques avec antagonisme d'effet entre un agoniste (morphine, méthadone) et un agoniste partiel (buprénorphine, nalméfène) ou un antagoniste (naloxone) ;

- par action au niveau de deux systèmes distincts, régulant le même effet (glycémie, kaliémie, natrémie, etc.).

\section{T2 Conséquences des interactions médicamenteuses}

\section{TEG1 Les conséquences délétères :}

- apparition ou majoration d'effets indésirables : sédatifs, anticholinergiques, sérotoninergiques, troubles ioniques, troubles de la glycémie, hypo/hypertension, abaissement du seuil épileptogène, saignements, torsades de pointes, etc. ;

- perte ou diminution d'efficacité : neuroleptiques et lévodopa, corticoïdes et antidiabétiques, AINS et antihypertenseurs, etc. ;

- syndrome de sevrage : association d'un agoniste (morphine, méthadone) à un agoniste partiel (buprénorphine, nalméfène...) ou un antagoniste (naloxone).

\section{TEG1 Les conséquences bénéfiques :}

- augmentation de l'efficacité thérapeutique : synergie bactéricide contre Pneumocystis carinii du sulfaméthoxazole (inhibe la synthèse d'acide folique) associé au triméthoprime (inhibe la réduction de la tétrahydrofolate réductase) ;

- diminution du risque d'effets indésirables : moindres variations de la kaliémie lors de l'association hydrochlorothiazide/IEC ou sartan ; 
- antidotes : flumazénil, antagoniste des récepteurs GABA permet de lever les signes de l'intoxication aux benzodiazépines, agonistes de ces mêmes récepteurs ;

- réduction du risque de mésusage : Suboxone ${ }^{\circledR}$, qui associe la buprénorphine à la naloxone, non absorbée per os, vise à limiter l'utilisation par voie intraveineuse (IV) des comprimés.

\section{T2 Conclusion}

Plutôt que d'espérer retenir par cœur des listes d'interactions pharmacodynamiques, mieux vaut, pour les reconnaître et les prévenir, identifier des groupes pharmaco-thérapeutiques susceptibles d'induire des effets indésirables et des situations ou des patients à risque (polymédication, âge, insuffisance rénale, facteurs environnementaux, automédication...). Bien connaître et analyser la pharmacologie des médicaments reste le meilleur moyen d'anticiper et/ou de gérer une telle interaction.

La base de données publique des médicaments et le Thesaurus des interactions médicamenteuses, récemment mis à jour et disponible sur le site l'ANSM, sont des outils précieux et rapidement accessibles en cas de doute sur une éventuelle interaction. Enfin, le Centre régional de pharmacovigilance dont dépend la pharmacie répond à toute question sur les médicaments et leurs interactions (encadré 1).

\section{Encadré 1. Retrouver les coordonnées du CRPV}

Pour retrouver les coordonnées du centre régional de pharmacovigilance (CRPV) dont l'officine dépend, il convient de consulter la carte interactive (www.rfcrpv.fr/contacter-votre-crpv/) ou le site de l'Agence nationale de sécurité du médicament et des produits de santé (ANSM) (http://ansm.sante.fr/Declarer-un-effet-indesirable/Pharmacovigilance/Centresregionaux-de-pharmacovigilance/(offset)/4).

\section{Points à retenir}

- Les interactions médicamenteuses relèvent de deux mécanismes : pharmacocinétique et pharmacodynamique.

- Les interactions pharmacocinétiques les plus préjudiciables sont celles qui amènent la concentration sanguine du médicament en dehors de l'intervalle thérapeutique. Plus cet intervalle est étroit, plus l'interaction est problématique.

- L'activité des enzymes du métabolisme des médicaments peut être augmentée (induction enzymatique) ou diminuée (inhibition enzymatique).

- Le site internet des Hôpitaux Universitaires de Genève (HUG) propose un document synthétique et régulièrement mis à jour qui répertorie les principaux inducteurs et inhibiteurs des cytochromes P450 et d'une protéine d'efflux, la P-glycoprotéine. 
- Les interactions pharmacodynamiques concernent des médicaments ayant des propriétés pharmacologiques communes, complémentaires ou antagonistes ou encore des effets indésirables communs ou opposés vis-à-vis d'un même système physiologique.

Sur 2 colonnes à droite après la puce de fin

\section{Références}

[1] Ministère des Solidarités et de la Santé. Base de données publique des médicaments. http://basedonnees-publique.medicaments.gouv.fr

[2] Agence nationale de sécurité du médicament et des produits de santé (ANSM). Thesaurus des interactions médicamenteuses. http://ansm.sante.fr/Dossiers/Interactionsmedicamenteuses/Interactions-medicamenteuses/(offset)/0

[3] Hôpitaux Universitaires de Genève (HUG). Interactions médicamenteuses, cytochromes P450 et Pglycoprotéine (Pgp). www.hugge.ch/sites/interhug/files/structures/pharmacologie_et_toxicologie_cliniques/a5_cytochromes_6_2. pdf

[4] Haute Autorité de santé (HAS). Caractéristiques du médicament. Atazanavir. www.hassante.fr/portail/upload/docs/application/pdf/2008-07/ct-5432 reyataz .pdf

Déclaration de liens d'intérêts

Les auteurs déclarent ne pas avoir de liens d'intérêts.

\section{Illustration}

pic_illus1

(C) Nik_Merkulov/stock.adobe.fr

Légende :

Les interactions médicamenteuses impliquant une transformation de la pharmacocinétique d'un médicament "victime" d'une seconde molécule sont les plus nombreuses.

Sur 2 colonnes en haut à droite en première page 